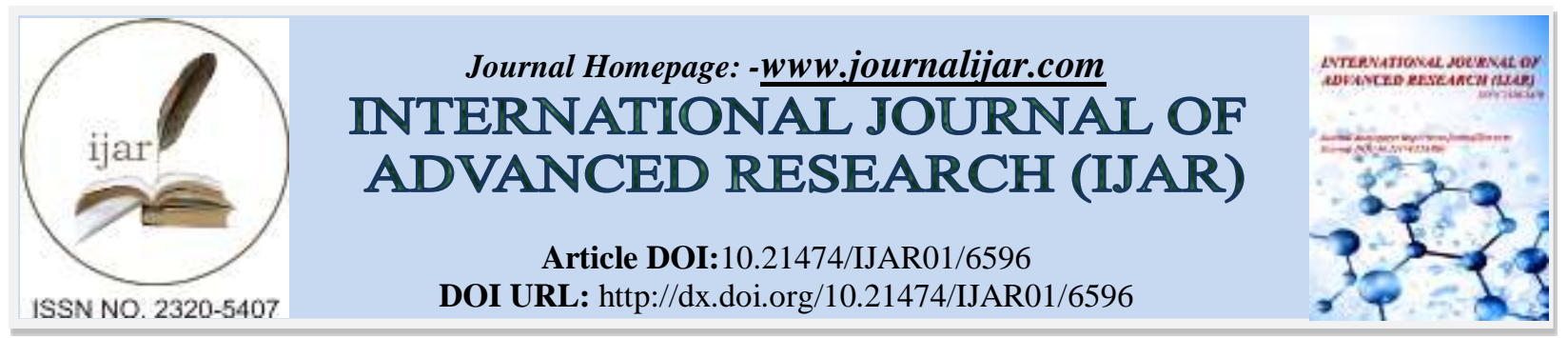

RESEARCH ARTICLE

\title{
A STUDY OF FLOPPY EYELID SYNDROME AND ITS OPHTHALMIC AND SYSTEMIC ASSOCIATIONS.
}

\section{Rupali Tyagi ${ }^{1}$, Varsha Kulkarni ${ }^{2}$ and Anjana Mirajkar ${ }^{3}$.}

1. Resident, Department of Ophthalmology Bharati Vidyapeeth University, Bharati Medical College, Hospital and Research Centre, Pune.

2. Professor, Department of Ophthalmology Bharati Vidyapeeth University, Bharati Medical College, Hospital and Research Centre, Pune.

3. Resident, Department of Ophthalmology Bharati Vidyapeeth University, Bharati Medical College, Hospital and Research Centre, Pune.

\section{Manuscript Info}

(..........................

Manuscript History

Received: 20 December 2017

Final Accepted: 22 January 2018

Published: February 2018

Keywords:-

Floppy eyelid syndrome, Glucoma, Obesity, Obstructive Sleep Apnea,

Superficial punctate keratopathy.

\section{Abstract}

Introduction: Floppy eyelid syndrome (FES) is characterized by very elastic upper lids that became easily distorted and evertible with minimal lateral traction and chronic papillary conjunctivitis of the upper palpebral conjunctiva. Apart from various ocular associations, FES has been associated with various systemic diseases like obesity, hypertension, IHD and DM. The present study was thus planned to gather data regarding the prevalence of FES and its ocular and systemic associations in the local population.

Materials \& Methods: A prospective non- randomized cross-sectional observational study was conducted at a tertiary care center including a total of 1600 cases. All cases were screened for floppy eye lid syndrome. Detailed history, ocular and systemic examination was conducted in all FES positive patients including screening for OSA. Results: Eighty one patients (5.06\%) out of 1600 screened were diagnosed to have FES. Prevalence of FES was more in males (71.6\%) as compared to females $(23.6 \%)$. Of the 81 patients detected to have FES, 57 were in the age group of 40-60 years. The commonest ocular involvement was superficial punctate keratopathy seen in $43.2 \%$ followed by tear film abnormalities seen in $37 \%$. The most common systemic association as determined by Berlin questionnaire was OSA (74.1\%).

Conclusion: Prevalence of FES in our study was 5.1\%. The commonest ocular association was superficial punctate keratopathy. Commonest systemic association was obstructive sleep apnoea. We thus recommend that screening for OSA should be undertaken in all patients of FES.

Copy Right, IJAR, 2018,. All rights reserved.

\section{Introduction:-}

Floppy eyelid syndrome (FES) was first described by Culbertson and Ostler in $1981^{1}$. It is an often underdiagnosed disorder of unknown pathogenesis. FES is characterized by very elastic upper lids that became easily distorted and 
evertible with minimal lateral traction and chronic papillary conjunctivitis of the upper palpebral conjunctiva and typically affects obese middle-aged men ${ }^{1}$. FES is often underdiagnosed or misdiagnosed because the symptoms of FES are similar to ocular surface disorder and concurrent presence of superficial punctate keratopathy and advanced Meibomian gland dysfunction may mislead the clinician ${ }^{2}$.

The prevalence of FES within the general population varies within a range of $2.3 \%$ and $3.8 \%{ }^{3}$. Various ocular pathologies have been associated to the syndrome like lash ptosis, blepharitis, upper lid entropion, lower lid ectropion medial and lateral canthal tendon laxity of upper and lower lids. The laxity of upper tarsal plate and spontaneous evertion during sleep leads to other ocular surface pathologies like chronic papillary conjunctivitis, superficial punctate keratopathy, corneal scarring, corneal neovascularisation, progressive epitheliopathy, or even corneal perforation. A high prevalence of keratoconus and glaucoma is reported in FES patients ${ }^{1,4-6}$.

FES has also been associated to a variety of systemic diseases as obesity, hypertension, ischemic heart disease, diabetes mellitus ${ }^{6}$. Some studies reported that there is close relationship between FES and Obstructive sleep apnea (OSA). These studies are based on the findings that both FES and OSA have similar pathologic changes in these two diseases, that is, a common defect in elastic tissue, and similar patient profiles, that is, middle-aged and obese men ${ }^{7}$. There are few studies reporting ocular and systemic associations of FES. Most of them are retrospective analysis of published data ${ }^{6}$.

A search of medical literature shows few reports on FES from India ${ }^{8}$. This study was thus planned to gather data regarding the prevalence of FES and its ocular and systemic associations in the local population.

\section{Materials And Methods:-}

A prospective non- randomized cross-sectional observational study was conducted at a tertiary care center including a total of 1600 Participants from Ophthalmology OPD and Pulmonary Medicine OPD of Bharati Hospital \& Research Center. All subjects were enrolled in the study after obtaining a written informed consent. Patients with history of previous lid surgeries, history of previous ocular trauma and any active infections of the eyes were excluded from the study.

Detailed history about ocular symptoms like itching, burning, decreased vision, discharge was taken. History of Systemic illness like diabetes mellitus, hypertension was recorded. History of Sleep habits like side they sleep on, snoring, frequent awakening was recorded. All 1600 patients were screened for floppy eye lid syndrome according to the following diagnostic criteria:

1. Lid eversion is maintained in downgaze for more than 6 seconds,

2. Associated with conjunctival papillary hypertrophy.

3. Spontaneous eversion of the upper lid after pulling it upwards.

All patients who were diagnosed of FES underwent a thorough ocular examination including:

1. Visual Acuity was done by Snellen's chart

2. Intra Ocular Pressure was taken by applanation tonometry

3. Anterior Segment

\section{LIDS:-}

1. Eversion test- Lax upper eyelid that was easily everted when pulled superiorly toward the eyebrow.

2. Medial canthal laxity- Pull the lower lid laterally, if there was punctal movement laterally more than $2 \mathrm{~mm}$ then it was considered positive. (FIGURE 2)

3. Lateral canthal laxity- Rounded appearance of lateral canthus.

4. Horizontal lid laxity

5. Distraction test: lower lid which could be pulled away from globe by $10 \mathrm{~mm}$ or more.

Snap back test:

1. Normal: lid "snaps back" quickly normal:

2. Mild laxity: slow return

3. Moderate laxity: incomplete return unless patient blinks

4. Severe laxity: incomplete return even after Blink

5. Ectropion 
Conjunctiva:- was carefully examined by slit lamp for any papillary hypertrophy, scarring and mucoid discharge.

\section{Cornea:-}

Was examined for superficial punctate keratitis by fluoroscein stain.

1. Any Opacities, vascularization, corneal ulcers.

2. Keratoconus was suspected in patients with high myopes with astigmatism more than $2 \mathrm{D}$ and was ruled out by slit lamp examination and keratometry.

Anterior Chamber:- was checked for any inflammatory reaction.

Pupils:- were examined for reaction.

Lens:- was examined for lenticular opacities.

Fundus Examination:- By 90D under full mydriasis by Tropicamide 0.5\% eye drops was done.

\section{Dry Eye Tests:-}

1. Tear breakup time (TBUT) was done to assess for evaporative dry eye disease.

2. Schirmers test: It was used for the assessment of aqueous tear production.

Glaucoma Suspect:- Patient was considered as glaucoma suspect

1. Intraocular pressure(IOP) of 22 or greater or

2. Cup disc $(\mathrm{CD})$ ratio 0.5 or greater

3. $\mathrm{CD}$ asymmetry ie $>$ or $=0.2$ difference between two eyes

4. IOP asymmetry ie $>$ or $=3 \mathrm{mmHg}$ difference with $\mathrm{IOP}>$ or $=16$

These suspects were further evaluated by complete glaucoma workup

Blood sugar levels:- random, fasting and post prandial levels were done.

Blood pressure:- of all patients was measured by sphygmomanometer in right brachial artery in supine position.

Body mass index (bmi):- is a measure of body fat based on height and weight that applies to adult men and women. Calculated by weight in kilograms $(\mathrm{kg})$ by height in meters squared $\left(\mathrm{m}^{2}\right)$ :

1. Underweight: $<18.5$

2. Normal weight: $18.5-24.9$

3. Overweight: $25-29.9$

4. Obesity: BMI of 30 or greater

\section{Obstructive Sleep Apnoea:-}

Screening was done by Berlin Questionnaire (ANNEX). All patients were subjected to Berlin questionnaire as a screening tool for OSA and those were positive were advised to undergo polysomnography.

\section{Results:-}

A total of 1600 patients of both gender above the age of 40 years were screened for FES. Eighty one patients (162 eyes) were diagnosed to have FES. These 81 patients were further evaluated for ocular pathologies and systemic evaluation for BMI, diabetes mellitus and systemic hypertension. Screening for OSA was as done by Berlin questionnaire and confirmed on polysomnography. Prevalence of FES was more in males (71.6\%) as compared to females (23.6\%). Of the 81 patients detected to have FES, 57 were in the age group of 40-60 years, 23 in the group $60-80$ years and only 1 patient was older than 80 years. The commonest ocular involvement was superficial punctate keratopathy seen in $43.2 \%(\mathrm{n}=69)$ followed by tear film abnormalities seen in $37 \%(\mathrm{n}=58)$. Other associations found were lower lid laxity $14.8 \%(n=24)$, glaucoma $(\mathrm{POAG}) 4.9 \%(\mathrm{n}=8)$, keratoconus $1.2 \%(\mathrm{n}=2)$ and lower lid ectropion 2.4\% $(\mathrm{n}=4)$ (Table 1). The most common systemic association as determined by Berlin questionnaire was OSA, 74.1\% $(\mathrm{n}=60)$. However, of these, 24.7\% $(\mathrm{n}=20)$ patients were positive on polysomnography as well. Other associations were obesity $25.9 \%(\mathrm{n}=21)$, systemic hypertension $22.2 \%(\mathrm{n}=18)$ and diabetes mellitus $18.5 \%(\mathrm{n}=15)$ (Table 2). 
Table 1:- Distribution of patients according to ocular associations

\begin{tabular}{|l|c|c|}
\hline \multicolumn{1}{|c|}{ OCULAR } & N & \%age \\
\hline LOWER LID LAXITY & 24 & $14.8 \%$ \\
\hline ECTROPION & 4 & $2.4 \%$ \\
\hline KERATOCONUS & 2 & $1.2 \%$ \\
\hline SPK & 69 & $43.2 \%$ \\
\hline TEAR FILM ABNORMALITY & 58 & $37 \%$ \\
\hline GLAUCOMA (POAG) & 8 & $4.9 \%$ \\
\hline
\end{tabular}

Table 2:- Distribution of patients according to systemic associations

\begin{tabular}{|l|c|c|}
\hline Systemic Manifestations & N (n-81) & \% \\
\hline OSA & 60 & $74.1 \%$ \\
\hline BMI $\left(>30 \mathrm{Kg} / \mathrm{m}^{2}\right)$ & 21 & $25.9 \%$ \\
\hline HYPERTENSION & 18 & $22.2 \%$ \\
\hline DIABETES & 15 & $18.5 \%$ \\
\hline
\end{tabular}

\section{Discussion:-}

FES is more prevalent in males than in females. The reported prevalence in other studies ranges from $56 \%$ to $89 \%{ }^{4-}$ 6 . In this study also the prevalence of males was more (71.6\% males and $23.6 \%$ females). FES is commonly reported in elderly patients. The common age group reported in most of the studies was 40-60 years ${ }^{5}$. In our study maximum patients were in the age group of 40-60 years. In our study, all patients had bilateral involvement with unequal severity. Culbertson reported more severe symptoms on the side of which patient sleeps. This may be due to rubbing of the eye against pillow ${ }^{4}$.

The reported prevalence of lower lid laxity ranges between $6 \%$ to $35 \%{ }^{5,6}$. Lower lid laxity was present in $14.8 \%$ of our patients. In a study by Daniel Ezra, lower lid abnormalities like ectropion, medial and lateral canthal tendon laxity were reported in $6 \%$ of cases ${ }^{5}$. Lower lid ectropion was seen in $2.4 \%$ of the patients in our study. In FES the upper tarsal plate is of rubbery consistency and gets folded on itself very easily. It is proposed that the repeated eye rubbing and stress cause up regulation of elastolytic enzymes like matrix metalloproteinase 7 and 9 . There is loss of tarsal elastin leading to floppiness of the upper lid. This is also responsible for lash ptosis ${ }^{9}$. Similar pathological changes may occur in lower lid tarsal plate and the canthal tendons causing vertical and horizontal lower lid laxity. Severe laxity manifests as ectropion.

In 1994 Culbertson WW and Tseng SC ${ }^{4}$ reported corneal abnormalities in $71 \%$ of their cases. Most common was superficial punctate epithelial keratopathy (45\%). Severity was more on the side on which the patient is habituated to sleep. Superficial punctate keratopathy was reported in $45 \%$ cases by Amy Fowler et.al. ${ }^{6}$. Daniel Ezra et al. reported 4 cases of microbial keratitis ${ }^{5}$. In our study, SPK was present in $43.2 \%(n=69)$ patients. None of our patients had microbial keratitis, filamentary keratitis, scarring or neovascularization of cornea. Our results were comparable with these studies.

It is postulated that the floppiness of the upper lid and spontaneous eversion during sleep compromises the distribution of the tear film over cornea and conjunctiva ${ }^{10}$. The chronic papillary conjunctivitis and meibomian gland dysfunction further affects the corneal epithelium leading to superficial punctuate keratopathy. The keratopathy is diffuse and involves entire cornea. Chronic insult along with tear film abnormality can cause filamentary keratitis. Scarring and superficial neovascularisation of the cornea is also reported in cases of FES.

FES leads to an improper closure of the eye. The poor contact between lid and the globe leads to inadequate spreading of tear film. The mechanical trauma and chronic inflammation of meibomian glands also cause lipid tear abnormalities. Histopathological changes in Meibomian glands like cystic degeneration, squamous metaplasia of orifice and atrophy of meibomian gland ducts are reported in cases of FES ${ }^{11}$.

Liu DT et al. studied the tear film dynamics in 16 patients of FES. Lipid Tear film abnormalities were found in $75 \%$ of the eyes. The tear evaporation rates and evaporation of lid skin rates were higher in patients of FES. They studied the lipid layer spread based on kinetic analysis of tear interference images indicative of lipid tear deficiency ${ }^{12}$. Amy Fowler et al. reported, higher incidence of dry eyes $4.5 \%$ in patients with FES as compared to that in general 
population i.e. $1.4 \%^{6}$. It was retrospective analysis of published cases so there may lack of documentation underestimating the prevalence of dry eye. Tear film abnormalities were present in $37 \%(\mathrm{n}=58)$ of our patients detected by TBUT, indicating unstable tear film. Schirmer's tests results were normal suggesting adequate aqueous tear secretion. The prevalence of dry eye was higher in our study group. This may be explained by the fact that the prevalence of dry eye is more in India than in western countries ${ }^{13}$.

It is proposed that eye rubbing may be a causative factor in keratoconus and possibly also FES. The reported prevalence of keratoconus in FES patients ranges from $7 \%^{5}$ to $59.80 \%{ }^{6}$. This wide range may be because of difference in assessment techniques. Culbertson and Tseng reported $10 \%$ prevalence of keratoconus ${ }^{4}$. Fowler AM reported 7\% prevalence of keratoconus in their retrospective analysis ${ }^{5,6}$. Daniel G Ezra et al. evaluated 102 diagnosed cases of FES ${ }^{5}$. Objective assessment of keratoconus was done using Oculus intruments Pentacam Schiempflug photographic camera system. They reported keratoconus in 61(59.8\%) cases out of 102 cases of FES. Whereas keratoconus was seen in 6 cases out of 102 controls $^{5}$. In our study, the incidence was $1.2 \%(\mathrm{n}=2)$. Our evaluation consisted of slit lamp examination and keratometry. More cases may have been detected by Oculus Pentacam Schiempflug photographic camera system.

There are no studies reporting prevalence of open angle glaucoma in cases of FES. There is a strong association of FES with OSA and a higher prevalence of primary open angle glaucoma and normotensive glaucoma in patients is well documented in the literature ${ }^{14}$. MA Jesus Muniesa, Manuel Sanchez -de-la-Torre e al reported a high prevalence of $(23.0 \%)$ glaucoma in OSA patients having FES. Whereas the prevalence of glaucoma in OSA patients without FES was only $5.33 \%{ }^{14}$. They proposed that in patients with OSA, there are prolonged repetitive periods of apnoea accompanied by transient hypoxia followed by re- perfusion. This ischaemia re-perfusion theory is supposed to play an important etiological role in FES. Similar changes may contribute to optic nerve changes and thereby glaucomatous changes ${ }^{14}$. In our study, $4.9 \%(\mathrm{n}=8)$ cases were diagnosed to have primary open angle glaucoma. All these patients also had OSA as confirmed on polysomnography which corresponded to the above study. It is advisable to screen all the patients with FES for primary Open Angle Glaucoma and normotensive glaucoma.

As per, Culbertson and Ostler's hypothesis ${ }^{1}$, there could be a subtle generalised connective tissue alteration related to eyelid laxity in FES. Elastin fiber depletion and collagen fibres changes in oropharyngeal tissue may lead to OSA. The prevalence of OSA in India is $13.7 \%{ }^{15}$. Amy fowler reported the prevalence of OSA in $21 \%$ patients of FES $^{6}$.Other studies also observed a significant association between FES and OSA ${ }^{5}(32 \%)$. In our study, the most common systemic association with FES was OSA as per Berlin questionnaire. However polysomnography confirmed the diagnosis in only $20(24.7 \%)$ patients. Our study results were comparable with other studies. All FES patients should be adviced for sleep studies preferably polysomnography to rule out OSA.

In a case series published by Culbertson and Ostler, all 11 patients of FES were obese males ${ }^{1}$. In series of 60 patients Culbertson and Tseng only 29\% patients with FES were obese ${ }^{4}$. In a study conducted by Daniel Ezra et al., 102 patients with FES showed that obese men in their sixth decade were more commonly affected $(37 \%)^{5}$. In a study by Amy fowler, obesity was reported to be present in $52 \%$ FES patients ${ }^{6}$. Panagiotis G Beis et al, conducted a study to investigate the association of FES with BMI. They did not find any significant association ${ }^{16}$. Though, they found hyperelasticity of the lids in the patients with high BMI, it was not statistically significant. Incidence of obesity in rural population of various states in India ranges from 7.8 to $15.9 \%{ }^{17}$. In our study, high BMI was recorded in $25.9 \%$ patients. It corresponded with the study by Culbertson and Tseng et al. ${ }^{4}$.

In a study by Daniel Ezra, systemic hypertension was diagnosed in $42 \%$ cases of FES and $16 \%$ cases had diabetes mellitus ${ }^{5}$. In our study, diabetics were $18.5 \%$ and hypertensives were $22.2 \%$. The reported prevalence of diabetes mellitus in the age group of above age of 40 years is around $9.3 \%$ in west India ${ }^{18}$. Overall prevalence of systemic Hypertension is $29.8 \%$ in India ${ }^{19}$ There was no significant association of systemic hypertension and diabetes mellitus with FES in our study patients.

\section{Conclusion:-}

Prevalence of FES in our study was 5.1\%. The commonest ocular association was superficial punctate keratopathy. Commonest systemic association was obstructive sleep apnoea. We thus recommend that screening for OSA should be undertaken in all patients of FES. 


\section{Conflict Of Interests:-}

The authors declare that there is no conflict of interests regarding the publication of this paper.

\section{Acknowledgments:-}

The authors gratefully acknowledge great help received from the scholars whose articles are cited and included in references of this paper. The authors are also grateful to authors/ editors/ publishers of all those articles, journals and books from where the literature for this paper has been reviewed and discussed.

\section{References:-}

1. W. W. Culbertson and H. B. Ostler, "The floppy eyelid syndrome," American Journal of Ophthalmology. 1981;92(4):568-75.

2. Karpecki PM, Shechtman DL. FES: a diagnostic challenge: patients who present with floppy eyelid syndrome are frequently misdiagnosed. Here's why. Review of Optometry. 2013 Aug 15;150(8):76-8.

3. Wang P, Yu DJ, Feng G, Long ZH, Liu CJ, Li H, Zhao TL. Is Floppy Eyelid Syndrome More Prevalent in Obstructive Sleep Apnea Syndrome Patients?. Journal of ophthalmology. 2016 Jun 5;2016.

4. Culbertson WW, Tseng SC. Corneal disorders in floppy eyelid syndrome. Cornea. 1994 Jan 1;13(1):33-42.

5. Ezra DG, Beaconsfield M, Sira M, Bunce C, Wormald R, Collin R. The associations of floppy eyelid syndrome: a case control study. Ophthalmology. 2010 Apr 30;117(4):831-8.

6. Fowler AM, Dutton JJ. Floppy eyelid syndrome as a subset of lax eyelid conditions: relationships and clinical relevance (an ASOPRS thesis). Ophthalmic Plastic \& Reconstructive Surgery. 2010 May 1;26(3):195-204.

7. McNab AA. Floppy eyelid syndrome and obstructive sleep apnea. Ophthal Plast Reconstr Surg 1997;13:98114.

8. Abhijit Das, MD,DM corresponding author and Ashalatha Radhakrishnan,MD, DM. Teaching NeuroImages: Floppy eyelids in obstructive sleep apnea syndrome. Neurology. 2011;77(22): 130-131.

9. Schlotzer-Schrehardt U, Stojkovic M, Hofmann-Rummelt C, Cursiefen C, Kruse FE, Holbach LM. The pathogenesis of floppy eyelid syndrome: involvement of matrix metalloproteinases in elastic fiber degradation. Ophthalmology. 2005;112(4):694-704.

10. Parunovic A. Floppy eyelid syndrome. Br J Ophthalmol. 1983;67(4):264-6.

11. Gonnering RS, Sonelland PR. Meibomiam gland dysfunction in floppy eyelid syndrome. Ophthal Plast Reconstr Surg. 1987;3(2):99-103.

12. Liu DT, Di Pascuale MA, Sawai J, Gao YY, Tseng SC. Tear film dynamics in floppy eyelid syndrome. Investigative ophthalmology \& visual science. 2005 Apr 1;46(4):1188-94.

13. Gupta N, Prasad I, Himashree G, D'Souza P. Prevalence of dry eye at high altitude: a case controlled comparative study. High Alt Med Biol 2008; 9: 327-34.

14. M. Muniesa, M. Sánchez-De-La-Torre, V. Huerva, M. Lumbierres, and F. Barbé, "Floppy eyelid syndrome as an indicator of the presence of glaucoma in patients with obstructive sleep apnea," Journal of Glaucoma, vol. 23, no. 1, pp. e81-e85, 2014.

15. Sharma SK, Kumpawat S, Banga A, Goel A. Prevalence and risk factors of obstructive sleep apnea syndrome in a population of Delhi, India. CHEST Journal. 2006 Jul 1;130(1):149-56.

16. Beis PG, Brozou CG, Gourgoulianis KI, Pastaka C, Chatzoulis DZ, Tsironi EE. The floppy eyelid syndrome: evaluating lid laxity and its correlation to sleep apnea syndrome and body mass index. ISRN ophthalmology. 2012 Jun 20;2012.

17. Mumbai: IIPS; 2007. International Institute for Population Sciences (IIPS) and Macro International. National Family Health Survey (NFHS-3), 2005-06: India: vol.I.

18. Kaveeshwar SA, Cornwall J. The current state of diabetes mellitus in India. The Australasian medical journal. 2014;7(1):45.

19. Anchala R, Kannuri NK, Pant H, Khan H, Franco OH, Di Angelantonio E, Prabhakaran D. Hypertension in India: a systematic review and meta-analysis of prevalence, awareness, and control of hypertension. Journal of hypertension. 2014 Jun;32(6):1170. 